\title{
Fetal acoustic stimulation test for early intrapartum fetal monitoring
}

\author{
M Goonewardene ${ }^{1}, K_{\text {Hanwellage }}^{2}$ \\ (Index words: fetal acoustic stimulation test, intrapartum fetal monitoring)
}

\begin{abstract}
Introduction The fetal acoustic stimulation test (FAST) is a simple cost effective screening test for antenatal fetal monitoring. The aim of the study was to evaluate the FAST as a screening test for early intrapartum fetal well being.
\end{abstract}

Methods An initial non stress test (NST) followed by a FAST using corometric model 146 was carried out in 486 participants in early labour with uncomplicated singleton pregnancies and $>32$ weeks gestation. A repeat NST was recorded in the participants who had an initial non reactive NST. The results of the NST and FAST were compared with fetal outcome. Maternal perception of fetal movements after FAST, results of NST before and after FAST, and the babies' 5 minute APGAR scores were measured.

Results Of the 486 participants 413 (85\%) noticed fetal movements after FAST. Initial NST was non reactive in $203(42 \%)$ but $149(31 \%)$ became reactive after FAST. Compared to the NST, FAST had a better sensitivity $(97 \%$ vs $62 \%, p<0.001)$, specificity $(100 \%$ vs $87 \%, p=0.017)$, positive predictive value $(100 \%$ vs $98 \%, p=0.024)$, negative predictive value $(79 \%$ vs $17 \%, p<0.001)$ and accuracy $(99 \%$ vs $64 \%, p<0.001)$ in predicting 5 minute APGAR $<7$ in the baby.

Conclusions FAST is a reliable screening test for assessing fetal well being in early labour. It complements the NST and is better than the NST alone.

Ceylon Medical Journal 2011; 56: 14-18

\section{Introduction}

In 1986, researchers in Singapore demonstrated that cardiotocography (CTG) on admission to hospital, which was referred to as the 'admission test', was a good screening test for fetal distress in labour [1]. Subsequent studies have not shown an improvement in neonatal outcome with routine use of the admission test [2,3] and therefore it is not recommended as a routine procedure [4]. However, it could be carried out as an 'insurance policy' that permits the non use of CTG for another 3-4 hours [5].

Vibro acoustic stimulation of the fetus, referred to as the fetal acoustic stimulation test (FAST), can convert a false positive (non reactive) non stress test (NST) to a reactive NST and also shortens the time duration that a NST needs to be carried out [6,7]. Furthermore the FAST complements and improves antenatal fetal biophysical profile assessments $[8,9]$. Therefore the FAST is used for antenatal fetal monitoring in several centers worldwide [5-9].

The FAST has also been shown to be useful for assessment of fetal wellbeing during the early intrapartum period [10-12]. It has been shown that fetuses subjected to the FAST react with fetal heart rate accelerations if they are not acidotic [11-13]. Auditory sensation is considered to be the first to be affected by hypoxia and a negative response to the FAST may indicate hypoxia [13]. Studies conducted to assess the safety of the FAST have not demonstrated any short or long term adverse effects on the fetus [14-16].

The authors of a recent Cochrane Systematic Review have recommended further research in to vibro acoustic stimulation for fetal assessment during labour [17]. In a previous study we showed that the FAST was a reliable and cost effective method of screening for antenatal fetal wellbeing, which could be used to complement the NST [18]. The objective of the current study was to evaluate the FAST as a screening method for assessing fetal well being in the early intrapartum period.

\section{Methods}

This study was conducted from 1 August to 24 December 2004 and 28 March to 30 April 2005 at Teaching Hospital, Mahamodara, Galle and from 9 May to 31 July 2005 at Castle Street Hospital for Women, Colombo (the study had to be temporarily suspended from 25 December 2004 to 27th March 2005 on account of the tsunami). Approval was obtained from the Ethical Review Committee of The Faculty of Medicine, University of Ruhuna. Consecutive women $(n=486)$ with uncomplicated singleton pregnancies at periods of gestation $>32$ weeks, establishing spontaneous onset of labour with contractions occurring at intervals of 3-10 minutes were recruited, after obtaining informed written consent. A 20 minute NST was recorded and was documented as reactive or nonreactive using the standard criteria [19]. Soon after the NST, a FAST was performed with the mother in the semi-recumbent position. The vibro acoustic stimulator

${ }^{1}$ Department of Obstetrics and Gynaecology, Faculty of Medicine, University of Ruhuna, Galle and ${ }^{2}$ University Obstetrics and Gynaecology Unit, Teaching Hospital, Mahamodara, Galle, Sri Lanka.

Correspondence: MG, e-mail <malikg@eureka.lk>. Received 24 May and revised version accepted 25 September 2010. Competing interests: none declared. 
was applied firmly to the maternal abdomen directly overlying the fetal thorax and the mother was asked whether she felt any movements. A kick immediately following the sound stimulation was marked as "movements". If no fetal movements were felt within five seconds the stimulation was repeated twice within 30 seconds. If no fetal movements were experienced during the three stimulations, the test was marked as "no movements". A repeat NST was carried out in the participants who had initial nonreactive NST before the FAST. Neither the FAST nor the NST were included in the routine management of singleton uncomplicated pregnancies at $>32$ weeks gestation in the units. Therefore, without reference to the NST and FAST results, all participants were allowed to progress in labour with intermittent auscultation of fetal heart sounds and subsequent CTG carried out only if considered necessary as per the unit policies prevailing at the time. Augmentation with oxytocin infusion and or amniotomy was carried out when indicated and the routine methods of detecting early fetal distress (intermittent auscultation combined with external cardiotocography when considered necessary) were adopted. The routine and appropriate management of any detected fetal distress, was carried out. The mode of delivery, presence or absence of meconium at delivery, and the 5 min APGAR score of the babies were documented.

The vibro acoustic stimulator used for the study was Corometric $^{\mathrm{TM}}$ model 146 , a dry battery powered device and having an audio frequency of $75 \mathrm{~Hz} \pm 10 \%$ sound intensity of $74 \mathrm{db}$ at one meter in air, and a maximum stimulation duration of 3 seconds \pm 0.5 seconds. NST was recorded using SONICAID ${ }^{\mathrm{TM}}$ Team MMC TG 003 cardiotocography recorder.

\section{Results}

The mean age of the 486 participants was 28.8 years
(SD 4.9). The majority (54.7\%) were primigravidae. The mean period of gestation (POG) of the participants was 38.8 weeks SD 1.4 and $39(8 \%)$ had a POG of $>41$ weeks.

Of the 486 women, 413 (85\%) felt fetal movements after FAST and $203(42 \%)$ had non reactive NST before the FAST but 150 (31\%) were false positives as they had a reactive NST after the FAST. Of the 486 women 43 (9\%) continued to have a non reactive NST and did not feel any fetal movements even after the FAST (Table 1).

Of the 43 women who continued to have nonreactive NST after the FAST, 36 needed emergency intrapartum caesarean delivery as they developed fetal heart rate abnormalities indicating intrapartum hypoxia. At delivery all 36 had meconium stained liquor and all the babies' APGAR scores were $<7$. The two primigravidae and five multigravidae who delivered vaginally had non asphyxiated babies but all seven had meconium stained liquor.

Of the 486 babies delivered, $39(8 \%)$ were asphyxiated and 6 of these mothers had felt fetal movements after the FAST (false negative FAST $=15 \%$ ). Of the 447 non asphyxiated babies delivered, 40 of the mothers also did not feel fetal movements after the FAST (false positive FAST $=9 \%$ ) (Table 2).

All women $(n=39)$ who delivered asphyxiated babies had non reactive NST which persisted even after the FAST. However, 14 of the women who had persistent nonreactive NST after the FAST delivered non asphyxiated babies (Table 3 ).

In the prediction of neonatal asphyxia, the NST had a better sensitivity than the FAST $(p<0.001)$ and the FAST had a better specificity than the NST $(p<0.001)$. The specificity, the positive predictive value and the accuracy of the NST were better $(p<0.001)$ after the FAST (Table 4$)$. The likelihood ratio of a positive NST was markedly increased after the FAST.

Table 1. FAST and NST results $(\mathbf{n}=\mathbf{4 8 6})$

\begin{tabular}{|c|c|c|c|c|c|}
\hline & \multicolumn{2}{|c|}{ NST before FAST } & \multicolumn{3}{|c|}{ NST after FAST } \\
\hline & Nonreactive & Reactive & Nonreactive & Reactive & Total \\
\hline No movements & 51 & 22 & 43 & 30 & 73 \\
\hline Movements $^{+}$ & 152 & 261 & 10 & 403 & 413 \\
\hline Total & 203 & 283 & 53 & 433 & 486 \\
\hline
\end{tabular}

NST $=$ Non stress test

FAST $=$ Fetal acoustic stimulation test 
Table 2. FAST results and 5 minute APGAR scores $(n=486)$

\begin{tabular}{lcc}
\hline & \multicolumn{2}{c}{ FAST } \\
\cline { 2 - 3 } & No movements & Movements + \\
\hline APGAR $<7$ & 33 & 6 \\
APGAR $>7$ & 40 & 407 \\
Total & 73 & 413 \\
\hline
\end{tabular}

FAST $=$ Fetal acoustic stimulation test

Table 3. NST before and after FAST and 5 min. APGAR scores $(n=486)$

\begin{tabular}{ccccc}
\hline & \multicolumn{2}{c}{ NSTbefore FAST } & \multicolumn{2}{c}{ NSTafter FAST } \\
\cline { 2 - 4 } & Nonreactive & Reactive & Nonreactive & Reactive \\
\hline APGAR $<7$ & 39 & 0 & 39 & 0 \\
APGAR $>7$ & 164 & 283 & 14 & 433 \\
Total & 203 & 283 & 53 & 433 \\
\hline
\end{tabular}

NST $=$ Non stress test

FAST $=$ Fetal acoustic stimulation test

Table 4. Ability of FAST and NST to predict neonatal asphyxia (APGAR $<7$ )

\begin{tabular}{lccccc}
\hline & FAST & NST before FAST & $p$ & NSTafter FAST & $p$ \\
\hline Sensitivity & $84.6 \%$ & $100 \%$ & $<0.001$ & $100 \%$ & \\
Specificity & $91.1 \%$ & $63.3 \%$ & $<0.001$ & $96.9 \%$ & $<0.001$ \\
PPV & $45.2 \%$ & $19.2 \%$ & $<0.001$ & $73.6 \%$ & $<0.001$ \\
NPV & $98.5 \%$ & $100 \%$ & 0.155 & $100 \%$ & \\
& & & $(\mathrm{NS})$ & & \\
Accuracy & $90.5 \%$ & $66.3 \%$ & $<0.001$. & $97.1 \%$ & $<0.001$ \\
LR Pos & 9.4 & 2.7 & & 100 & \\
LR Neg & 0.17 & 0 & & 0 & \\
\hline
\end{tabular}

$\mathrm{PPV}=$ Positive predictive value

NPV $=$ Negative predictive value

LR Pos = Likelihood ratio of a positive test

LR Neg $=$ Likelihood ratio of a negative test

$\mathrm{NS}=$ Not significant 


\section{Discussion}

A nonreactive CTG per se does not indicate fetal hypoxia and therefore it does not warrant a caesarean section [CS]. In fact because a non reactive CTG has a very poor specificity and a very high false positive rate, it can lead to unnecessary CS with no benefit to neonates. Therefore a routine CTG on admission to the labour room is not recommended for low risk patients $[2,3,4]$.

In the absence of a fetal heart rate abnormality which would indicate possible intrapartum hypoxia, the routine intrapartum management as per the unit policies prevailing at the time, were continued after the initial assessment with CTG and FAST. The cessation of fetal breathing movements at the onset of labour is a well known phenomenon considered as an early diagnostic sign of the onset of labour. The value of the presence or absence of fetal movements after a FAST and the ability to convert a false positive nonreactive CTG to a reactive CTG thereby increasing the specificity of a CTG during early labour, were the factors being evaluated in the present study. Therefore the Ethical Review Committee of the Medical Faculty of the University of Ruhuna approved the research protocol which stipulated that the routine management of the unit should be followed disregarding the FAST outcome, the value of which has not been established and which was the objective of the study.

The FAST has a similar predictive value to the oxytocin challenge test (OCT) which was described several years ago [5,20,21]. However, the FAST is easier, simpler, quicker and safer than the OCT especially with an overdistended or scarred uterus. The FAST by itself can have significant false positive and false negative results and therefore should not be used alone [22,23]. In the present study the NST had a $100 \%$ sensitivity and the combination of the NST and the FAST had a 96.9\% specificity in predicting neonatal asphyxia (APGAR $<7$ ).

Although seven women who continued to have nonreactive NST after the FAST and felt no fetal movements after the FAST delivered vaginally, all had meconium stained liquor which was not observed at amniotomy or spontaneous rupture of membranes during early labour.

The presence of meconium could also indicate late gestation. However, as there was no meconium staining of liquor in any of these patients when amniotomy was carried out in early labour after the initial assessment with the CTG and FAST, the subsequent appearance of meconium suggests subsequent occurrence of some degree of intrapartum hypoxia.

Thirty six (84\%) of the women who had a nonreactive NST continued to have a nonreactive NST even after the FAST, and did not feel any fetal movements after the FAST. All of them needed emergency CS for fetal distress, and all their babies were asphyxiated at birth (APGAR <7). There were no neonatal deaths nor significant neonatal morbidity in the 43 babies who were asphyxiated (APGAR
$<7)$ at subsequent delivery by CS. All had 10 min APGAR scores of $>7$ and all of them were well in 3-5 days.

With a reactive NST, the chance of having an asphyxiated baby is virtually zero (likelihood ratio of a negative NST $=0$ ). In the presence of a non reactive NST the addition of the FAST will predict fetal hypoxia in virtually all cases (likelihood ratio of a positive NST after FAST $=100$ ). Therefore it is useful to introduce an admission test with the NST and the FAST to routinely screen for hypoxia in the early intrapartum period.

\section{Conclusion}

The combination of the NST and the FAST is a good method to screen for hypoxia in the early intrapartum period.

\section{References}

1. Ingermasson I, Arulkumaran S, Ingermasson E, Tambyraja RL, Ratnam SS. Admission test a screening test for fetal distress in labour. Obstetrics and Gynaecology 1986; 68: 800-6.

2. Impey L, Reynolds M, Mac Quillan K, Gates S, Murphy J, Sheil O. Admission cardiotocography: a randomised controlled trial. Lancet 2003; 361: 465-70.

3. Bix E, Reiner LM, Clovning A, Oipan P. Prognostic value of the labour admission test and its effectiveness compared with auscultation only: a systematic review. British Journal of Obstetrics and Gynaecology 2005; 112: 1595-604.

4. National Institute for Health and Clinical Excellence (NICE). The use of electronic fetal monitoring: the use and interpretation of cardiotocography in intrapartum fetal surveillance. NICE inherited clinical guideline C. London: NICE; 2007. Available online; http:// Guidance.nice.org. uk/ CGC/niceguidance/html. accessed 24 April 2010.

5. Gibb D, Arulkumaran S. The admission test by cardiotocography or by auscultation. In: Fetal Monitoring in Practice 3rd ed. Butterworth Heinemann, Elsevier, London 2008: 103-22.

6. Saracoglu F, Gol K, Sahin I, Turkkani B, Oztopcu C. The predictive value of fetal acoustic stimulation. Journal of Perinatology 1999; 19: 103-5.

7. Tan KH, Symth R. Fetal vibro acoustic stimulation for facilitation of tests for fetal wellbeing. Cochrane Database of Systematic Reviews 2001; (1); CD002963;

8. Tongson T. Piyamongkol W, Anantachote A. The rapid biophysical profile for assessment of fetal wellbeing. Journal of Obstetrics and Gynaecology Research 1999; 25: 431-6.

9. Kumar SA. Vibro acoustic stimulation and modified fetal biophysical profile in high risk pregnancy. Journal of Obstetrics and Gynaecology of India 2007; 57:37-41.

10. Chittacharoen A, Srijhantuik K, Suthutvoravut S, Herabutya Y. Maternal perception of sound provoked fetal movements in the early intrapartum period. International Journal of Gynaecology and Obstetrics 1997; 57: 129-33.

11. Chauhah SP, Hendrix NW, Devoe LD, Scardo JA. Fetal acoustic stimulation in early labour and pathological fetal 
hypoxia: a preliminary report. Journal of Maternal and Fetal Medicine 1999; 8: 208-12.

12. Lin CC, Vassallo B, Mittendorf R. Is intrapartum vibroacoustic stimulation an effective predictor of fetal acidosis? Journal of Perinatal Medicine 2001; 29: 506-12.

13. Gibb D, Arulkumaran S. The role of scalp pH. In: Fetal Monitoring in Practice. 3rd ed Butterworth Heinemann, Elsevier, London 2008: 198-202.

14. Fisk NM, Nicolaidis PK, Arulkumaran S, Weg MW, Tannirandom Y, Nicolini U, Parkes MJ, Rodeck CH. Vibroacoustic stimulation is not associated with sudden fetal catecholamine release. Early Human Development 1991; 25: 11-7.

15. Arulkumaran S, Skurr B, Tong H, Kek LP, Yeoh KH, Ratnam SS. No evidence of hearing loss due to fetal acoustic stimulation test. Obstetrics and Gynaecology 1991; 78: 283-5.

16. Spencer JA, Deans A, Nicolaidis P, Arulkumaran S. Fetal heart rate response to vibroacoustic stimulation during low and high fetal heart rate variability episodes in late pregnancy. American Journal of Obstetrics and Gynaecology 1991; 165: 86-90.

17. East CE, Smyth RMD, Leader LR, Henshall NE, Colditz PB, Tan KH. Vibro acoustic stimulation for fetal assessment in labour in the presence of a non reassuring fetal heart rate trace. The Cochrane Database of Systematic Reviews 2005, issue (2): Article No: CD004664. DOI: 10.1002/14651858. pub 2.In: Cochrane Library 2009, Issue 1.

18. Batcha TM, Goonewardene IMR. The fetal acoustic stimulation test: a reliable and cost effective method of antepartum fetal monitoring. The Ceylon Medical Journal 2005; 50: 156-9.

19. Arulkumaran S, Symonds EM. Intrapartum fetal monitoring basic knowledge. The Obstetrician and Gynaecologist 1999; 1: $12-4$.

20. Schiff E, Lipitz S, Sivan E, Barkai G, Mashiach S. Acoustic stimulation as a diagnostic test: comparison with oxytocin challenge test. Journal of Perinatal Medicine 1992; 20: 275-9.

21. Devoe LD. Antennal assessment; contraction stress test, non stress test, vibro acoustic stimulation, amniotic fluid volume, biophysical profile and modified biophysical profile - an overview. Seminars on Perinatology 2008; 32: 247-52.

22. Irion O, Stuckelberger P, Moutquin JM, Morabia A, Extermann $\mathrm{P}$, Beguin F. Is intrapartum vibratory acoustic stimulation a valid alternative to fetal scalp $\mathrm{pH}$ determination? British Journal of Obstetrics and Gynaecology 1996; 103: 642-7.

23. Skupski DW, Rosenberg CR, Eglinton GS. Intrapartum fetal stimulation tests: a meta analysis. Obstetrics and Gynaecology 2002; 99: 129-34. 